\title{
Behaviour of Roofing Materials Facing to Micro-Organisms
}

\author{
Marielle Fassier $^{1}$, Céline Faugeron ${ }^{2}$, Vincent Gloaguen ${ }^{2}$, Céline Ducroquetz ${ }^{1}$, Olivier Dupont ${ }^{1}$ \\ ${ }^{1}$ Centre Technique de Matériaux Naturels de Construction, Paris, France \\ ${ }^{2}$ Laboratoire de Chimie des Substances Naturelles, Limoges, France \\ Email:m.fassier@ctmnc.fr
}

Received September 18, 2012; revised October 20, 2012; accepted October 29, 2012

\begin{abstract}
An accelerated water-streaming test was used to evaluate several roofing materials regarding their behavior to colonization by algae, by closely reproducing the phenomenon of natural biological soiling. A set of roofing materials with defined physical and chemical characteristics was thus investigated against the colonization by algae. Porosity, roughness and chemical composition showed to be factors of influence in the establishment of those micro-organisms.
\end{abstract}

Keywords: Building Materials; Fired Clay Products; Water-Streaming Test; Micro-Organisms; Colonization

\section{Introduction}

Algae and cyanobacteria are the first colonizers of the sheathing of the buildings in humid areas after bacteria which are omnipresent. In fact, algae and cyanobacteria colonize all the surfaces where humidity is high. So they develop in marine area but also on land substrates such as plants, rocks, grounds or even building materials [1]. These micro-organisms are present in the air in the form of spores, cells or filaments and have, in case of enough lights, humidity and nutriments, the capacity to adapt their morphology and their physiology to colonize the different types of products quoted above.

On buildings, the accumulation of micro-organisms is translated by the presence of greener black spots in continental area and by red spots in coastal area [2-5]. These spots appear most of the time near the outlet pipes, the architectural flaws, and the fallout of drainpipes or the ventilation of conditioning.

Beside an unaesthetic aspect, the presence of microorganisms on the buildings causes physical and chemical damage to the materials. In fact, algae and cyanobacteria present on the surface of the material can cause for example the dissolution of the carbonate of calcium in the case of buildings made in stone or in concrete [5]. Except this case, the presence of micro-organisms on the surface of buildings promotes the penetration of the water in the porous network of the material which leads, first, to a inflating and the appearance of cracks and then to an increase of the humidity rate in the material which is propitious to the acceleration of the process of colonization [1].
At the present time, there are different means to fight against the micro-organisms such as the mechanical cleaning, the chemical cleaning or the hydrofugation. However, these treatments are, most of the time, toxic for the environment, not very effective and very expansive.

Algae and cyanobacteria form a series of varied species of unicellular and pluricellular plants which size is generally between $0.5 \mu \mathrm{m}$ and $1 \mathrm{~mm}$. These organisms are photo-autotrophe and need an environment vivid in light and humidity useful to their photosynthesis reaction.

Green algae, presented on building, are eukaryote organisms which the wall is composed of a rigid fibrillaire part and of a mucilagineuse part whereas cyanobacteria are prokaryote organisms composed of a wall formed by peptidoglycans and lipoproteins.

The colonization of the substrate by micro-organisms shows a dynamic aspect. Bacteria colonize quickly the surface of the material and they are followed by the establishment of algae and cyanobacteria. This establishment can be divided into five steps which are the followings: The development of an organic biofilm which absorbs ions and organic molecules at the surface of the material, the transport of micro-organisms on the surface and the formation of co-aggregates of micro-organisms, the reversible adhesion of micro-organisms and aggregates, the co-adhesion of micro-organisms each other and the irreversible adhesion of micro-organisms on the surface of material by the secretion of exo polymers [1]. This adhesion of micro-organisms is influenced by the topography, the chemical composition and the surface tension of the substrate.

Follow a seeding of the surface of building materials 
due to the uprooting, from a colonized substrate, of spores, cells or fragments of filaments and their transport, algae and cyanobacteria develop on very various surfaces [6]. The biological development of algae and cyanobacteria is influenced by several environmental factors such as light, humidity, pluviometry, outside temperature, wind, atmospheric pollution and vegetal environment [1] but also by the physical and chemical characteristics of the colonized material such as porosity, roughness, surface tension and $\mathrm{pH}$.

Researches on facades led during the twenty last years by Rindi and Gaylarde have allowed to list algae and cyanobacteria presented most of the time on buildings [1,7-9].

Concerning algae, Klebsormidium Flaccidum seems to be the most frequent with a presence rate of $55 \%$. It follows by Trebouxiaspp, Stichococcus Bacillaris, Chlorosarcinopsis Minoe, Chlorella cf Mir and Bilis which have respectively a presence rate of $49 \%, 46 \%, 28 \%$ and $27 \%$.

For the cyanobacteria, the most represented on facades is Cyanosarana Parthenonensis with a presence rate of 28\% followed by Chroococcus Lithophilus, Calothrix Pulvinata, Leptolyngbya Foveolarum, Gloeocapsa Sanguinea and Phormidium Vorium which respectively have a presence rate of $21 \%, 21 \%, 18 \%, 15 \%$ and $13 \%$.

As Klebsormidium Flaccidum is the specie of algae the most present on the surfaces of building materials, it has been withheld for this study. The physical, chemical and surface characteristics of the different roofing products have been determined. According to previous studies, these criteria could have an influence on the colonization of substrates by micro-organisms and that is why they have been retained. This study aims to confirm these observations and to establish correlations between the physical and chemical parameters of products and their ability to be colonized by micro-organisms.

\section{Experimental}

\subsection{Klebsormidium Flaccidum}

Klebsormidium Flaccidum is a filamentous algae hardwearing to area strongly dehydrated even if it grows, most of the time, in area which have a high humidity.

This algae is composed of cells to barred-shaped, forming long filaments slightly constricted at cross-walls, one parietal and cup-shaped chloroplast, covering about $2 / 3$ of the cells, with one clearly visible pyrenoid $(L=5$ $25 \mu \mathrm{m}, \mathrm{w}=4-12 \mu \mathrm{m})[10,11]$.

The culture of Klebsormidium Flaccidum is realized in a modified Bold's basal medium composed of three mother solutions individually pressure-sealed, after their preparation, at $120^{\circ} \mathrm{C}$ for 20 minutes. 1 liter of liquid culture medium is composed of $10 \mathrm{~mL}$ of solution $\mathrm{A}, 10$ $\mathrm{mL}$ of solution $\mathrm{B}$ and $1 \mathrm{~mL}$ of solution trace introduced in 1 liter of ultra-pure water. The medium is adjusted at $\mathrm{pH} 7$ and then pressure-sealed before the introduction of the strain of algae [6].

Each Erlenmeyer flask is put on magnetic agitator, to oxygenate again the medium to have a better reproducetion and to homogenize the suspension, in anair-conditioned culture room at $20^{\circ} \mathrm{C} \pm 0.5^{\circ} \mathrm{C}$ where the light intensity is controlled (white light) and the photoperiod is $16 \mathrm{~h} / 8 \mathrm{~h}$ (light/darkness).

The dosage of algae contained in the 2 liters Erlenmeyer flasks is determined by the measurement of the dry mass of the algae. A known volume of algae suspension is filtered on membranes in micro fibers of glass which have a porosity of $1,2 \mu \mathrm{m}$ (Whatman GF/C). These membranes are dried at $105^{\circ} \mathrm{C}$ for 3 hours. Then, the dry algae mass is determined and attributed to the filtered volume.

\subsection{Analysis}

\subsection{1. pH Measurement}

Before $\mathrm{pH}$ measurement, materials are grinded to ob- tain powders which are dispersed in pure water and shaken with a magnetic agitator. This measurement is realized with a pHmeterCyberscan pH 1100 (Eutech instruments).

\subsubsection{Roughness Measurement}

The surface of a bright or not material is not a per- fectly polished surface. In fact, it presents a lot of micro or macro geometric irregularities which are defined by comparison with an average line and are classified into two categories: peak and hollow. All these surface flaws form the roughness.

The surface roughness can be determined by the measurement of some parameters such as:

- Rp: The maximal peak observed on the analyzed length;

- Rc: The maximal hollow observed on the analyzed length;

- Rt: The total roughness. It corresponds to the total of the maximal peak and the maximal hollow observed on the analyzed length;

- Ra: The average distances difference between successive peaks and hollows. It corresponds to the difference between the average distance and the average line.

When visual and tactile comparisons are not sufficient to distinguish a difference of roughness between two surfaces, it is necessary to use a roughness device. This device is composes of an induction transducer which has a diamond point. The variations in the generated tensions are located by a sensor and then converted in different roughness parameters by the electronic of the device.

This measurement has been realized with a roughness 
device TR 110 TIMe (Phynix). The obtained value is the average of fifty measurements realized on a sample of the substrate.

\subsubsection{Contact Angle Measurement}

Among the techniques used to determine the surface tension of a liquid put on a solid the measurement of the contact angle of the solid is the technique the most used. This technique consists in putting a drop of a liquid on the surface of a solid and in reading the angle between this drop and the substrate. The measured value allows going back to the surface energy of the solid due to the Young equation:

$$
\gamma_{\mathrm{SV}}=\gamma_{\mathrm{SL}}+\gamma_{\mathrm{LV}} \cos \theta
$$

where $\gamma_{\mathrm{LV}}$ is the superficial tension of the liquid in presence of vapour, $\gamma_{\mathrm{SL}}$, the interfacial tension between the solid and the liquid, $\gamma_{\mathrm{SV}}$, the superficial tension of the solid in presence of liquid and $\theta$, the contact angle between the liquid and the solid surface.

The experimental device used is composed of a light source and of a digital camera allowing taking a photograph of the drop. The damping angle is measured with software from digitized pictures. The drop is put on the solid surface with a syringe. The horizontality of the substrate is precisely adjusted. The software used allows digitizing the outline of the drop by the analysis of the pictures taken by the digital camera. To conclude, this software determines the static contact angle, of the liquid on the surface, thanks to interpolation methods by polynomials or by arcs of the circle.

\subsubsection{Porosity Measurement}

The determination of the density with a helium pycnometer is realized like that: the device precisely measures the variation of gaseous volume caused by the introduction of the sample in a calibrated enclosure. The volume is calculated from the pressure transfer observed when the gas passes from the cell containing the sample (pressure $\mathrm{P}_{2}$ ) to the reference cell (pressure $\mathrm{P}_{1}$ ). This method is based on the Mariotte's law.

$$
\mathrm{V}_{\text {ech }}=\mathrm{V}_{\text {cell }}-\mathrm{V}_{\text {exp }} /\left(\mathrm{P}_{1} / \mathrm{P}_{2}-1\right)
$$

The volume of the cell, $\mathrm{V}_{\text {cell }}$, and the expansion volume $\mathrm{V}_{\text {exp }}$ are constants. The maximal accuracy is $\pm 0.1 \%$ on the volume and $\pm 0.001 \mathrm{~g}$ on the weighing.

Then, the density is given by the relation:

$$
\rho=\frac{m}{\mathrm{~V}}
$$

where $\mathrm{m}$ is the sample mass and $\mathrm{V}$, the sample volume.

This measure has been realized with a helium pycnometer ACCUPYC 1330 (Micromeritics). From the densities of the bulkyand the grinded sample, the opened porosity has been deduced to use the equation:

$$
\mathrm{P}=\left(\rho_{\text {(grinded) }}-\rho_{(\text {bulky })}\right) / \rho_{\text {(grinded })}
$$

\subsubsection{The Water-Streaming Test}

To evaluate the behavior of building materials facing with the presence of micro-organisms, an experimental device has been developed. This device simulates speeding up the colonization, by algae, on the exposed surface of materials.

The principle of the water-streaming test consists in the dampening of the surface of the material by water streaming which is comparable to the rain flow on facades or roof tiles of buildings.

This device, developed by Boulon and Dubosc, is composed of a closed box where the humidity is relatively high, favorable to the development of micro-organisms [12]. In this box, the samples are put back to back on two supports in stainless steel at an angle of $45^{\circ}$ to increase the colonization process. At regular time intervals, the samples are sprayed by the KlebsormidiumFlaccidum culture, kept at a constant temperature by a thermo-regulator, presented in the bottom of the box. This spraying is realized by a ramp pierced by holes which have a constant diameter at equal distance each other. Moreover, the box is installed in a room where the temperature and the relative humidity are controlled and have two footlights composed of two neon tubes which have a known intensity (1500 lux, $30 \mathrm{~W}$ ) and according to a photo period controlled (12 h/12 h).

At regular time intervals, the biological development is evaluated by pictures analysis. The kinetic of colonization on the surface of materials is evaluated by pictures analysis. The surface of the samples installed in the water-streaming test is digitized at regular time intervals with an office scanner. Then, the digitized pictures are treated to digitize the signal as on the Figure 1.

Then, the digitized pictures are treated with a pictures

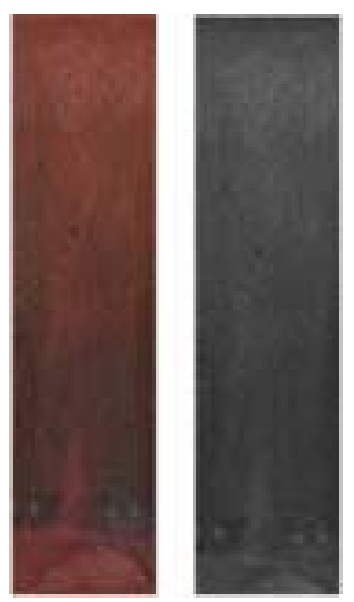

Figure 1. Example of a digitized and converted into 256 grey levels roof tile. 


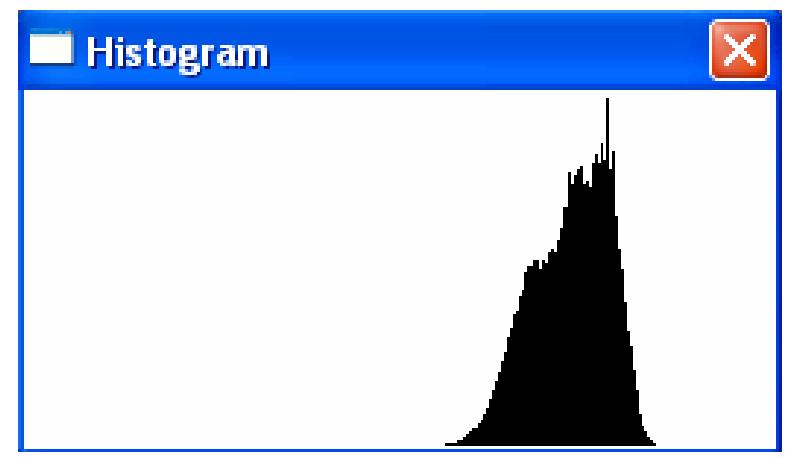

Figure 2. Histogram of the digitized picture representing the number of pixels in function of their intensity.

analysis program to establish ahistogram (Figure 2) representing the number of pixels in function of their intensity.

In this treatment, the intensity 0 is attributed to the black pixels and the white pixels correspond to the intensity 255 . At the end, to make the area corresponding to the colonization of the micro-organisms more detectable, a threshold is realized for the maximal intensity of the peaks of the histogram. So, the micro-organisms seem to be white on the black support. Then, the percentage of colonization on the sample is obtained making the ratio between the pixels corresponding to the micro-organisms and the total number of pixels. To the end, a graph showing the evolution of the growing of micro-organisms in function of the exposure time in the water-streaming test is drawn for each sample series.

\subsection{Materials}

During this study, the materials used are the followings:

Slate: It is a metamorphic rock belonging to the family of clayey shales. It is generally composed of silica, alumina, iron oxide, magnesia, potash and soda. It is hardwearing and its color can vary from the white to the black. Slate can be rectangular or scale-shaped. Its thickness varies between 3 and $9 \mathrm{~mm}$.

Concrete: It is a powdery material, making with water or a saline solution, a binding agent able to agglomerate varied substances when it hardens. This hydraulic bonding agent hardens quickly and reaches in few days its maximal resistance. So, after hardening, this paste keeps its resistance and its tenacity even under water. A homogenous mixture is realized by humid, half-humid, half dry or dry way and then the mixture is introduced in a tubular rotary kiln, lightly sloped, at a temperature about $2000^{\circ} \mathrm{C}$. A sudden and fast cooling of the mixture leads to the elaboration of the clinker. The composition of the concretes is a mixture of calcium silicates and calcium aluminates due to the combination of the lime with silica, alumina and iron oxide.
Glass: Glass is a hard, fragile, brittle and transparent to the visible radiation material, most of the time derived from siliceous sand. This is an amorphous material having an important structural disorder and the phenomenon of vitreous transition. It is generally composed of silica and flux. This material has good compatibility with most of chemical compounds exception to hydrofluoric acide.

Fibrocement: It is a building material composed of concrete reinforced by synthetic or natural fibers.

Clay roofing tiles: A roof tile is, most of the time, a fired clay product used as a roofing element. Fired clay products are made from fired clays at high temperature. So, the firing temperature is between $800^{\circ} \mathrm{C}$ and $1150^{\circ} \mathrm{C}$ to confer to the products a low porosity, a mechanical resistance, functionality and a surface aspect. The surface aspect depends of several parameters such as the choice of the raw materials, the drawing of the pattern and the process of fabrication. These products can be without coating, with a silicone coating or with a glazed coating.

\section{Results and Discussion}

\subsection{Physical and Chemical Characteristics of the Roofing Products}

The physical, chemical and surface characteristics of the different roofing products have been determined and summed up in the Table 1. These characteristics are the porosity, the contact angle, the roughness and the $\mathrm{pH}$.

Thanks to the porosity of the roofing products and particularly to their porosity rate, it is possible to divide the roofing products into two categories. In fact, fired clay roofing tiles, which have or not a silicone coating, show a high porosity such as the concrete tiles. The noticed porosity is between $15 \%$ and $33 \%$. Concerning the fibrocement sheets they have also a high porosity about $30 \%$. Contrary, the glass tiles and the slates show a low porosity widely inferior to the previous products because it does not exceed $1 \%$.

Concerning the contact angle of the surface of these different roofing products, it is very different from one

Table 1. Physical, chemical and surface characteristics of the roofing products.

\begin{tabular}{ccccc}
\hline Material & $\begin{array}{c}\text { Porosity } \\
(\%)\end{array}$ & $\begin{array}{c}\text { Contact angle } \\
\left({ }^{\circ}\right)\end{array}$ & $\begin{array}{c}\text { Roughness } \\
(\mu \mathrm{m})\end{array}$ & $\mathrm{pH}$ \\
\hline $\begin{array}{c}\text { Fired clay tile } \\
\text { without coating }\end{array}$ & 14.9 & 48.5 & 5.1 & 8.4 \\
$\begin{array}{c}\text { Fired clay tile with } \\
\text { a silicone coating }\end{array}$ & 29.4 & 67.5 & 4.4 & 8.2 \\
Concrete tile & 33.0 & 0.0 & 7.9 & 11.6 \\
Glass tile & 0.5 & 30.0 & 2.5 & \\
Slate & 0.7 & 20.0 & 3.8 & \\
Fibrocement & 29.9 & 0.0 & 6.8 & 9.9 \\
\hline
\end{tabular}


product to another but stays however in the area of hydrophilicity. In fact, the values of the static contact angle of a drop of water deposited on the surface of the different materials does not exceed $68^{\circ}$ which means that all the products cannot prevent water to stagnate at their surface provoking its streaming. So, these materials are propitious to their colonization by micro-organisms due to the fact of the presence of water at their surface. However, it seems that when a water drop is deposited on the surface of a concrete tile or on a fibrocement sheet, a state of total damping is observed. This lets to think that these materials will be more favorable to the colonization by micro-organisms then the others and so the colonization will be faster and higher.

The average roughness of the surface of the different products is spread between 2.5 and $7.9 \mu \mathrm{m}$. The difference between the values is not very important but the higher value observed for the concrete tile lets to think that this product will be easier colonized by the microorganisms than the others and precisely than the glass tile which has the average roughness lower.

Concerning the $\mathrm{pH}$ values, they show that all the products have a basic surface which is propitious to their colonization by micro-organisms. In fact, algae are inclined to hang on substrates which have $\mathrm{pH}$ values between 9 and 11 [1]. These observations let to think that concrete tile, having a $\mathrm{pH}$ value about 11.6 , such as the fibrocement sheet $(\mathrm{pH} \approx 9.9)$, are more propitious to the colonization by micro-organisms than the others. So the colonization of their surface by algae will be faster and higher.

\subsection{Behavior of the Roofing Materials Facing with Micro-Organisms}

Concerning the roofing products, trials have been realized to compare their resistance to micro-organisms. The results are presented on Figure 3.

First, it is important to notice that whatever the coating

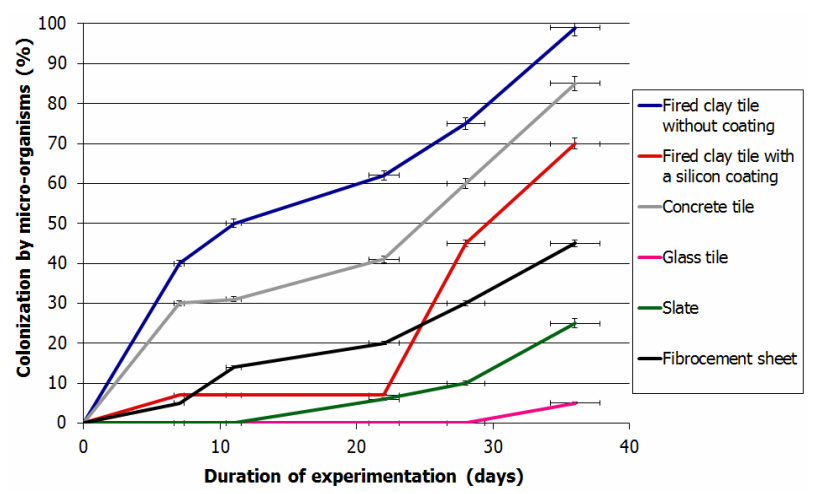

Figure 3. Kinetic of colonization and of growth of microorganisms on the surface of three samples of several roofing materials. or the material, the colonization of the surface by microorganisms does not appear after the same duration passed in the time passed in the water-streaming test. For the samples of fired clay tile without coating, between the 1 st and the 9th day, the colonization and the proliferation of micro-organisms on the surface of the samples is very high and very important. In fact, the surface of the samples is covered at $57 \%$ after nine days of exposure. This development is linear with regard to the exposure time during this period. Between the 9th and the 20th day, the proliferation of algae keeps accelerating becoming extremely important. After twenty days of exposure in the water-streaming test, the surface of the samples of fired clay tile is covered at $61 \%$. Between the 20th and the 32 nd day of exposure, the speed of colonization of micro-organisms strongly reduces to become independent of time. In fact, the rate of colonization becomes nearly constant to reach a total covering of the samples of $99 \%$ at the end of the experience. To conclude, the fired clay tile shows a fast and important greening.

For the samples of fired clay tile with a silicone coating, between the 1st and the 7th day, the percentage of colonization of the surface of the samples is about $7 \%$ which means that the colonization and the proliferation of micro-organisms is very slow. Micro-organisms have difficulties to hold on the substrate and to develop. Between the 7th and the 22nd day, the proliferation of micro-organisms stays constant which means that the proliferation of micro-organisms is stopped and that any new algae find hanging point at the surface of the samples. Then, between the 22nd and the 28th day, the proliferation of the micro-organisms strongly and quickly increases to reach a percentage of colonization of the surface about $45 \%$. So, algae find many hanging points which allow their development. This can be explained by the deterioration of the silicone layer located at the surface of the fired clay tile. In fact, after about twenty days of exposure in the water-streaming test, the silicone layer is damaged by the streaming of water and the fired clay tile with a silicone coating retakes the aspect and the properties of a fired clay tile without coating. Moreover, these results are in harmony with the release tests and the contact angle measurements realized on this product which detects a degradation of the silicone layer after 15 to 20 days of soaking. Finally, between the 28th and the 36th day, the proliferation of micro-organisms continues to be very important and fast. In fact, after 36 days of exposure, the surface of the samples is colonized at $70 \%$. This result is comparable to the result obtained with the fired clay tile without coating taking the delay of greening due to the silicone layer into account. To conclude, the colonization and the proliferation of micro-organisms on the surface of fired clay tile with a silicone coating is very slow and light during the first twenty two days of 
the experiment. The micro-organisms do not find hanging points, they cannot develop and they are washed by the successive watering. Silicone products, showing a hydrophobic surface, promote water streaming on their surface and so the slowdown in the colonization of surfaces by micro-organisms during this period. However, after twenty days of exposure, the silicone layer at the surface of samples is damaged by the successive water streaming and the behavior of the surface of the product becomes comparable to the behavior of a fired clay tile without coating. The colonization and the proliferation of micro-organisms become more important and the final covering rate of the samples reaches $70 \%$.

For the concrete tile, their behavior facing the greening is comparable to the behavior of a fired clay tile without coating. In fact, between the 1 st and the 7 th day, the colonization and the proliferation of micro-organisms at the surface of the samples are very high and very important. The surface of the samples is covered at $30 \%$ after seven days of exposure. This development is linear with regard to time exposure during this period. Between the 7 th and the 22 nd day, the proliferation of algae stays notable but the speed decreases with regard to the first period of the experiment. So, after twenty two days of exposure in the water-streaming test, the surface of the samples on concrete tile is covered about $41 \%$. Between the 22 nd and the 36 th day of exposure, the speed of colonization of micro-organisms strongly increases with regard to the exposure time. In fact, the colonization rate becomes very important and high to reach a final covering of the samples of $85 \%$ at the end of the experiment. To conclude, concrete tile shows a fast and important greening from the beginning of the experiment. Microorganisms find hanging and development points easily and they are uniformly distributed at the surface of the samples. The covering rate is important and reaches a final value of $85 \%$ of the surface.

For the fibrocement sheet, the kinetic of colonization of micro-organisms at their surface is comparable to the kinetic observed on fired clay tile without coating but the rate and the speed of proliferation of micro-organisms stay lower. So, between the 1 st and the 7 th day, the colonization and the proliferation of micro-organisms at the surface of the samples are limited and low. In fact, the surface of the samples is covered at 5\% after seven days of exposure. This development is linear with regard to the exposure time during this period. Between the 7 th and the 11th day, the proliferation of algae is so important and the development of algae at the surface of samples becomes faster and more present. So, after eleven days of exposure in the water-streaming test, the surface of samples is covered at $14 \%$. Between the 11 th and the 20th day of exposure, the speed of colonization of micro-organisms seems to stagnate and become independ- ent of the exposure time. Algae stay at the surface of the samples but do not grow. The colonization rate stays around $20 \%$ of the surface. Then, between the 22 nd and the 36th day of exposure, the colonization and the proliferation of micro-organisms at the surface of the fibrocement sheet continue and are very strong. The development of algae is faster and more important than in the previous period of the trial. The final covering of the samples is about $45 \%$. To conclude, the fibrocement sheet shows a more limited greening than the fired clay tile without coating at the beginning of the trial. However, the colonization of micro-organisms intensifies and becomes faster after about twenty days of exposure in the water-streaming test. So it seems that after a certain exposure time, the surface of the samples of fibrocement sheet is totally saturated with water and shows a biological layer and a nutritive medium propitious to the fixing and the development of micro-organisms. The final covering rate reaches $45 \%$ of the surface.

Concerning the slate, it is important to notice that the colonization by micro-organisms appears on these surfaces only after eleven days of exposure. Then, between the 11th and the 28th day, the colonization and the proliferation of micro-organisms at the surface of the samples are limited and low. In fact, the surface of the samples is covered at about $10 \%$ after twenty eight days of exposure. This development is linear with regard to the exposure time during this period. Between the 28th and the 36th day, the proliferation slightly increases but so significant and the development of algae at the surface of the samples becomes more important and present. So, after thirty six days of exposure in the water-streaming test, the surface of the samples is covered at about $25 \%$. To conclude, the samples of slate show an important delay of greening with regard of fired clay tile without coating. In fact, the greening appears only after eleven days of exposure in the water-streaming test. At the end of this period, algae develop at the surface of the samples but slowly and it is limited because the final covering after thirty six days of exposure does not exceed $25 \%$. So, these products show the property to delay so important the greening with regard to the fired clay product without coating thanks to, in majority, their physical, chemical and surface properties.

At the end, for the glass tile, it is important to notice that the colonization by micro-organisms appears on these surfaces only after twenty eight days of exposure. Then, between the 28 th and the 36 th day, the colonization and the proliferation of micro-organisms at the surface of the samples are limited and low. In fact, the surface of the samples is covered between $5 \%$ and $6 \%$ after thirty six days of exposure. This development is linear with regard to the exposure time during this period. To conclude, the samples of glass tile show an important 
delay of greening with regard to the fired clay tile without coating. In fact, the greening appears only after twenty eight days of exposure in the water-streaming test. Further to this period, algae develop at the surface of the samples but this development is slow and limited because the final covering after thirty six days of exposure dos not exceed $6 \%$. So these products show the property to delay so important the greening with regard to the fired clay tile without coating thanks to, in majority, their physical, chemical and surface characteristics.

\section{Conclusions}

The analysis of physical, chemical and surface characteristics of roofing products allows putting above differences between materials and doing supposition with regard to their behavior facing micro-organisms. The analysis of curves representing the percentage of colonization of these surfaces by micro-organisms allows confirming these suppositions.

In fact, it appears that:

- More the porosity is high, more the colonization of substrates, by micro-organisms, is fast and important;

- More the contact angle of the surface of the products is low, that is to say more water is inclined to spread on the support, more the colonization of substrates is fast and important;

- More the average roughness of the surface of the substrates is high, more the colonization of supports is fast and important;

- For basic pH values, micro-organisms hold on the substrates and develop easier at the surface of substrates.

For the future, it could be interesting to observe the behavior of the building materials in front of micro-organisms when they are treated with commercial hydrofuge solutions. This future study can be interesting to evaluate the efficiency of these commercial products against micro-organisms.

\section{REFERENCES}

[1] H. Barbebrousse, B. Ruot, C. Yéprémian and G. Boulon, "An Assessment of Façade Coatings against Colonisation by Aerial and Cyanobacteria," Building and Environment, Vol. 42, No. 7, 2007, pp. 2555-2561. doi:10.1016/j.buildenv.2006.07.031

[2] P. Berdahl, H. Akbari, R. Levinson and W. A. Miller, "Weathering of Roofing Materials-An Overview," Construction and Building Materials, Vol. 22, No. 4, 2008, pp. 423-433. doi:10.1016/j.conbuildmat.2006.10.015

[3] D. M. John, "Algae Growths on Buildings: A General Review and Methods of Treatment," Biodeterioration Abstracts, Vol. 2, No. 67, 1988, pp. 81-102.

[4] R. J. Lombardo, H. Barberousse, G. Tell and A. Couté, "Factors Involved in the Colonization of Building Facades by Algae and Cyanobacteria in France," Biofouling, Vol. 22, No. 2, 2006, pp. 69-77.

doi:10.1080/08927010600564712

[5] H. E. Schlichting, "Some Subaerial Algae from Ireland," British Phycological Journal, Vol. 10, No. 3, 1975, pp. 257-261. doi:10.1080/00071617500650251

[6] H. C. Bold, "Arbornealgae: Their Abundance and Heterogeneity," Science, Vol. 143, No. 3606, 1964, pp. 583585. doi:10.1126/science.143.3606.583

[7] C. C. Gaylarde, P. M. Gaylarde, J. Copp and B. Neilan, "Polyphasic Detection of Cyanobacteria in Terrestrial Biofilms," Biofouling, Vol. 20, No. 2, 2004, pp. 71-79. doi:10.1080/08927010410001681237

[8] F. Rindi and M. D. Guiry, "Composition and Distribution of Subarial Algal Assemblages in Galway City, Western Ireland," Cryptogamie-Algologie, Vol. 24, No. 3, 2003, pp. 245-267.

[9] F. Rindi and M. D. Guiry, "Composition and Spatial Variability of Terrestrial Algal Assemblages Occurring at the Bases of Urban Walls in Europe," Phycologia, Vol. 43, No. 3, 2004, pp. 225-235. doi:10.2216/i0031-8884-43-3-225.1

[10] M. A. Shirakawa, K. Loh, V. M. John, M. E. S. Silva and C. C. Gaylarde, "Biodeterioration of Painted Mortar Surfaces in Tropical Urban and Coastal Situations: Comparison of Four Paint Formulations," International Biodeterioration \& Biodegradation, Vol. 65, No. 5, 2011, pp. 669-674. doi:10.1016/j.ibiod.2011.03.004

[11] K. D. Stewart and K. R. Mattox, "Structural Evolution in the Flagellated Cells of Green Algae and Land Plants," Biosystems, Vol. 10, No. 1-2, 1978, pp. 145-152. doi:10.1016/0303-2647(78)90036-9

[12] A. Dubosc, G. Escadeillas and P. J. Blanc, "Characterization of Biological Stains on External Concrete Walls and Influence of Concrete as Underlying Material," Cement and Concrete Research, Vol. 31, No. 11, 2001, pp. 16131617. doi:10.1016/S0008-8846(01)00613-5 